SLAC-PUB-8633

October 2000

\title{
Photonic Band Gap Fiber Accelerator
}

Xintian Eddie Lin

Submitted to Physical Review Letters

Stanford Linear Accelerator Center, Stanford University, Stanford, CA 94309

Work supported by Department of Energy contract DE-AC03-76SF00515. 


\title{
Photonic Band Gap Fiber Accelerator
}

\author{
Xintian Eddie $\operatorname{Lin}^{\dagger}$ \\ Stanford Linear Accelerator Center, Stanford University, Stanford, CA 94309
}

(Dated: October 1, 2000)

\begin{abstract}
We present and analyze a conceptually new kind of charged particle accelerator, making use of a photonic band gap lattice for field confinement near the beam axis, and employing dielectric material to produce a synchronous longitudinal electric field. An example design is presented, we also discuss the absence of higher order dipole modes and ease of fabrication.
\end{abstract}

PACS numbers: 41.75.Jv, 42.70.Qs

There are three requirements in making an electromagnet accelerator: longitudinal electric field, synchronization and confinement. Traditional microwave accelerators can be viewed as metallic waveguide transporting $\mathrm{TM}_{01}$ mode down the tube. Periodic disk loading slows the phase velocity of the wave to the speed of light (SOL), at which the relativistic particles are traveling. This approach has serious limitations when we try to make accelerators at higher gradient. It is a result of the following scaling law.

From dimensional consideration, we can relate the accelerating gradient $E_{0}$ to the transported power $P$ by

$$
Z_{c}=\frac{E_{0}^{2} \lambda^{2}}{P}
$$

where $\lambda$ is the wavelength and the longitudinal impedance $Z_{c}$ is a functional of geometry and independent of length scale and loss (Including loss in transportation only limits the length of each accelerator structure.). This motivates higher frequency for higher gradient with attendant difficulties from stronger higher order modes and $3 \mathrm{D}$ machining. Another obstacle is the lack of good material to enclose the electromagnetic fields at higher frequency. For example, the attenuation length is $0.7 \mathrm{~m}-$ $\mathrm{m}$ for $1 \mu \mathrm{m}$ wavelength $\mathrm{TM}_{01}$ mode in a $2 \mu \mathrm{m}$ diameter copper tube. Here we propose a different kind of accelerator structure that addresses the aforementioned difficulties: It is based on dielectric waveguide and photonic band gap (PBG) artificial lattice.

It is well known that the wave in a hollow waveguide has phase velocity higher than the speed of light, while dielectric lining in the waveguide has the effect of slowing down the phase velocity. Field solution of this configuration is available[1]. In the case of dielectric constant $\epsilon_{r}=2.13$ and $a / b=0.55$ (Fig. 1), the SOL frequencies $k_{0}=\omega / c$ are $2.96,8.17,14.13$ for $\mathrm{TM}_{01}, \mathrm{TM}_{02}$ and $\mathrm{TM}_{03}$ modes respectively. Normalization to $1 / b$ is used. Field

\footnotetext{
†eddie@slac.stanford.edu
}

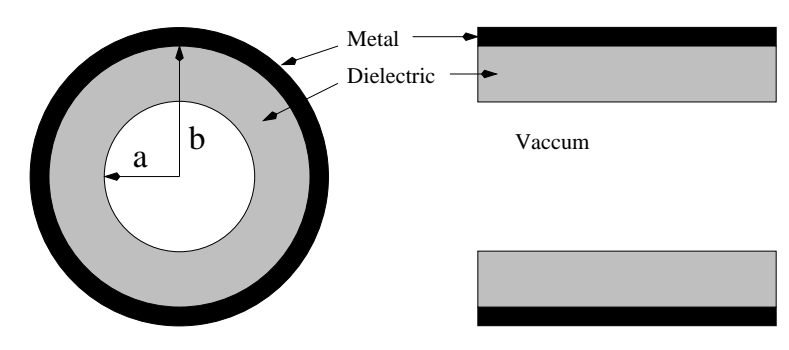

FIG. 1: Dielectric Waveguide Accelerator.

distribution is plotted in Fig. 2. From the material damage and wall loss point of view, $\mathrm{TM}_{01}$ mode is superior to other higher order monopole modes by virtue of its smaller magnetic field on the wall and smaller electric field in the dielectric.

Dielectric waveguide accelerators (DWA) have been tested in the laboratory at C-band $(7.8 \mathrm{GHz})[2]$ and

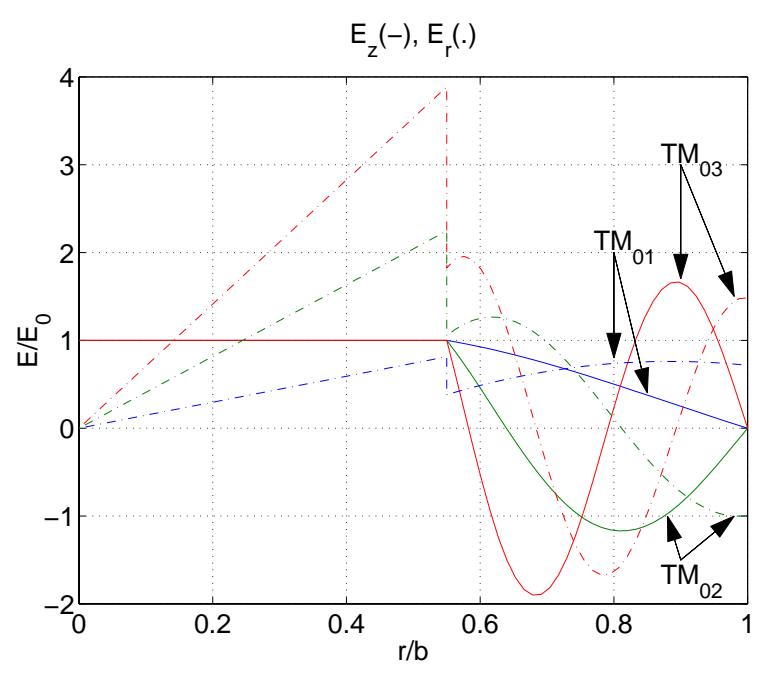

FIG. 2: Electric field distribution inside DWA. The Solid and dotted lines represent longitudinal and transverse electric fields, respectively. 
a planar version at $\mathrm{W}$-band $(91 \mathrm{GHz})$ has also been tested[3].

As we pointed out, metal at shorter wavelength is too lossy to be an effective material to confine and transport EM waves. On the other hand, PBG material, proposed by Yablonovitch[4] and John[5], is promising. A photonic crystal is a periodic structure in one, two or three dimensions. The scattering of the electromagnetic wave in the periodic lattice may give rise to a forbidden energy gap, over which the EM waves can not propagate. This phenomena is analogous to the band gap formation of semiconductor materials, where the photon wave function is replaced by electron wave function. A familiar example in one dimension is the multilayer coating on dielectric mirror. Because dielectric has extremely low loss, PBG material can replace metal as perfect reflector.

A simple approach is to replace the metal in Fig. 1 by a multilayer coating. Fink[6] etc. has demonstrated guiding in air with such a coating. Alternatively, one could replace the metal with two or three dimensional photonic crystal that has band gap at appropriate frequency. Single mode guiding in two dimensional close packed hexagonal lattice has been demonstrated in the visible over $40 \mathrm{~mm}[7]$. A three dimensional photonic crystal at infrared has also been fabricated on a 6-inch wafer[8]. We choose the two dimensional hexagonal lattice based on the manufacturing process and the material's high breakdown threshold, which will be discussed later.

A 2D PBG has a periodic structure in the $x-y$ plane and is uniform in the $z$ direction. Earlier studies $[9,10]$ concerned in-plane propagation, in which the wave has no variation in the $z$ direction. Recently, more studies[7, $11,12]$ have concentrated on using 2D PBG to transport light. But the application in accelerator needs one more condition: energy band gap at SOL $\left(k_{0}=k_{z}\right)$ phase propagation. Fig. 3 illustrates such band gaps in a particular 2D hexagonal lattice. The 2D PBG material is composed of silica and air. The background is fused silica (gray, $\epsilon_{r}=2.13$ ) with air rod (white) forming the triangular lattice. Maxwell's equations are solved by Plane Wave Method [13] with $\beta=k_{z} / k_{0}=1$. In the simulation 469 plane waves are used. The radius of the air $\operatorname{rod} r=0.35 a$, with $a$ the inter-rod distance. The lowest SOL band gap centers at $k_{0}=8.23$, where $k_{0}, k_{z}$ are normalized to $1 / a$. It is evident that there are several SOL band gaps. For accelerator applications, it would be ideal to have only one such gap that is utilized to trap the fundamental mode, and let all other higher order modes leak out. Such desirable PBG fiber guiding also depends on the formation of the defect.

The defect mode, introduced by making an air hole different from others, can be guided if the frequency is within the band gap. One realization is illustrated in Fig. 4. The frequency and field distribution are calculated by employing a supercell that includes the volume of $108 \mathrm{u}-$ nit cells. With the radius of the center hole $r_{0}=0.52 a$, the frequency of the defect mode, $k_{0}=k_{z}=8.20$, falls within the lowest SOL band gap. The mode is a $\mathrm{TM}_{0 n}$

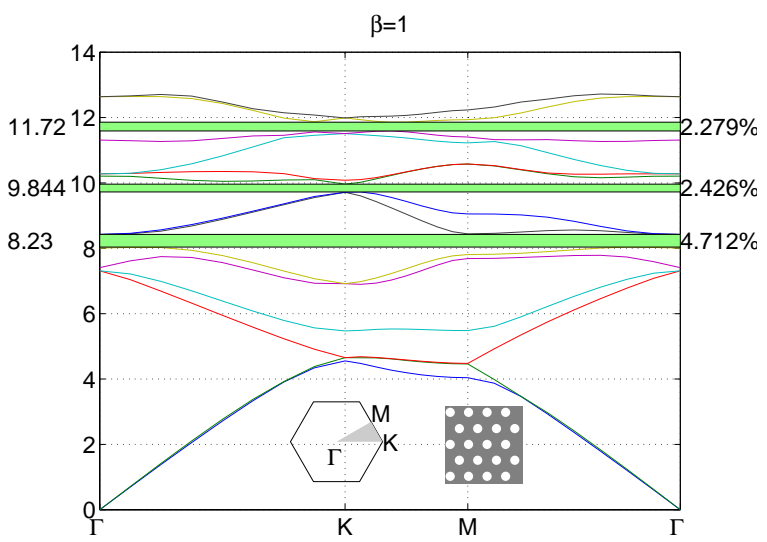

FIG. 3: Photonic band gaps in 2D. Band diagram is plotted over the irreducible Brillouin zone, highlighted in light gray. A section of the periodic structure is illustrated too, with silica in gray and air in white. The green regions in the plot are the band gaps. Midgap values and gap-midgap ratios are displayed on the left and right of the band, respectively.
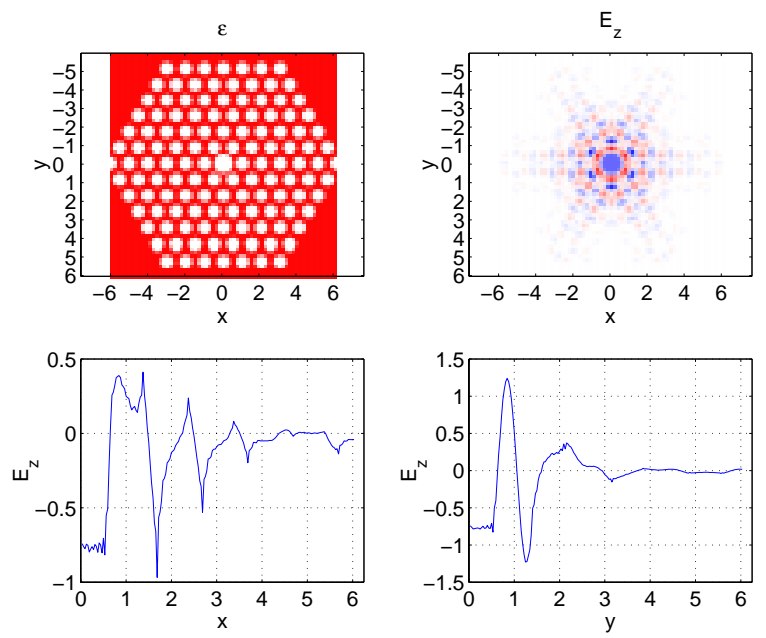

FIG. 4: The dielectric distribution and the field distribution of the defect mode trapped by 2D PBG fiber. In the top right plot, color coding, blue and red, represents negative and positive values respectively.

like mode with longitudinal electric field uniform inside the center defect. The rapid oscillation of $E_{z}$ inside the center defect is an artifact of finite number (18 per unit cell) of plane waves included in the calculation. The field outside the core decays steadily at about $6 \mathrm{~dB}$ per unit cell in the $x$ direction. Due to the large decay length, part of the PBG material acts as the dielectric in Fig. 1. A simple DWA model of one free parameter $a / b=0.55$ gives a synchronized $\mathrm{TM}_{02}$ mode at $k_{0}=8.17$ and $v_{g} / c=0.60$, which compare very well with the simulation: $k_{0}=8.20$ and $v_{g} / c=0.58$.

The dispersion of the defect mode is plotted in Fig. 5. 
The band gap is in green and SOL line is in red. The

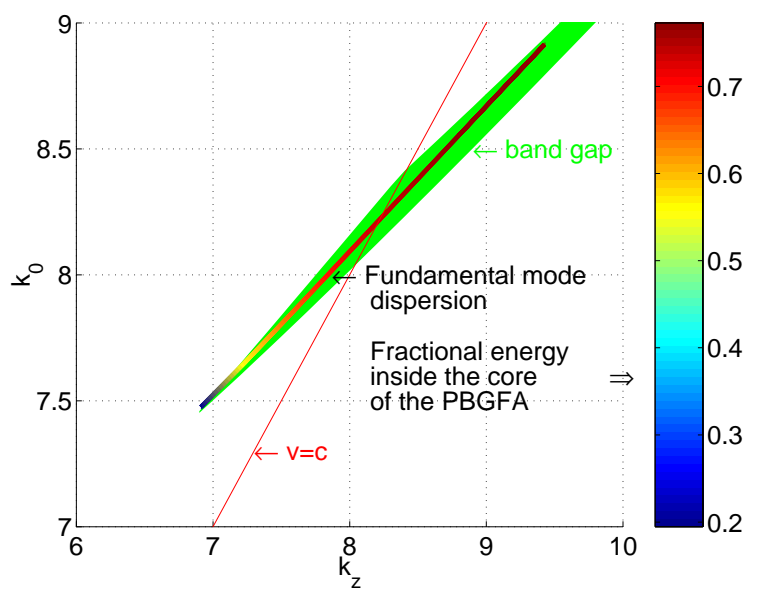

FIG. 5: The dispersion of the defect mode. The green band is the band gap responsible for localization. The dispersion curve is color coded by the energy fraction inside the core of the PBG fiber.

dispersion curve is color coded with the fractional energy inside the core of the PBG fiber. We artificially defined the core as the area within 2 lattice spacings of the center. In particular, the synchronized defect mode has about $73 \%$ of the stored energy inside the core, signifying a strongly trapped mode.

One interesting feature of electromagnetism in dielectric media is that there is no fundamental length scale other than the assumption that the system is macroscopic. Therefore, we are free to choose the wavelength that is convenient. One good starting point is at $\lambda=1$ $\mu \mathrm{m}$, where optical source and techniques are mature, and fused silica is relatively easy to manufacture and can withstand a high threshold fluence. Scaling provides design at other, perhaps more desirable wavelengths.

Because of the pulse shape slippage with respect to the relativistic beam, pulse length $\tau$ has to be longer than $\left(1-v_{g} / c\right) l / c$, where $l$ is the structure length. For a 10 $\mathrm{cm}$ long structure, $\tau>140 \mathrm{ps}$. At this pulse length, the damage threshold of $1.053 \mu \mathrm{m}$ laser is about $17 \mathrm{~J} / \mathrm{cm}^{2}$ on bulk fused silica[14], which translated to a peak electric field $E_{p}=0.79 \mathrm{GeV} / \mathrm{m}$. A square pulse is assumed. The simulation indicates that the peak electric field inside the dielectric material is 2.1 times that of the center electric field. Therefore the accelerating gradient will be limited to $E_{0}=0.38 \mathrm{GeV} / \mathrm{m}$. From the longitudinal impedance $Z_{c}=19.5 \Omega$ obtained in the simulation, we find out the power

$$
P=\frac{E_{0}^{2} \lambda^{2}}{Z_{c}}=7.4 \mathrm{~kW}
$$

needed to produce such field. At maximum energy trans-

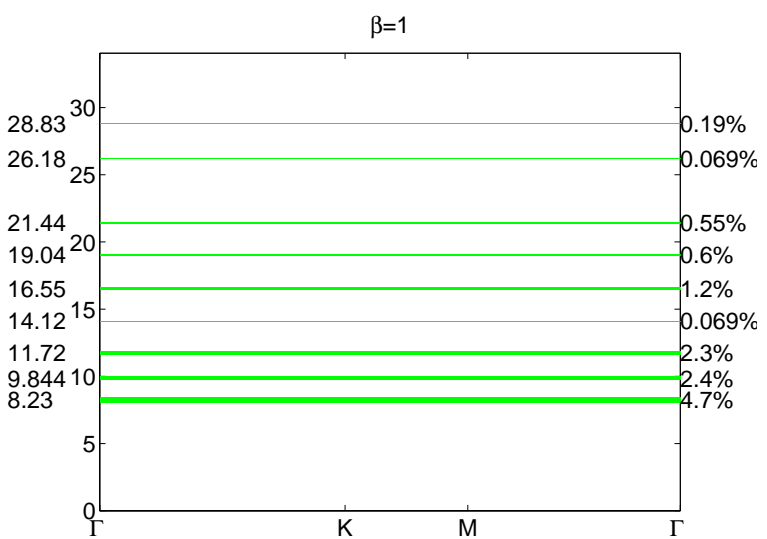

FIG. 6: Photonic band gaps within the lower 100 modes. For clarity, frequency bands are omitted.

fer, beam loaded gradient

$$
E_{0}^{l}=E_{0}-\frac{1}{4} \frac{q c Z_{c}}{\lambda^{2}} \frac{1}{1-v_{g} / c}
$$

equals half of unloaded gradient $E_{0}$. A point charge is assumed and fundamental theorem of beam loading[15] is applied. The maximum charge

$$
q_{m}=\frac{P \tau}{\frac{1}{2} E_{0} l}=\left(1-v_{g} / c\right) \frac{2 E_{0} \lambda^{2}}{Z_{c} c}
$$

corresponds to $3.4 \times 10^{5}$ electrons accelerated at a loaded gradient of $0.19 \mathrm{GeV} / \mathrm{m}$. It is worth noting that the laser power and thus maximum beam loading scales with wavelength to the second power, therefore more charge $\left(1.4 \times 10^{6}\right.$ electrons $)$ can be accelerated at $2 \mu \mathrm{m}$ wavelength. Due to extreme loss from IR absorption, wavelength longer than $2 \mu \mathrm{m}$ can hardly be transported in silica. To increase the charge, one can bundle an array of PBGFA to form a parallel accelerators or go to $10 \mu \mathrm{m}$ wavelength with chalcogenide glass.

Nonlinear effects, including self-phase modulation, Stimulated Raman and Brillouin Scattering are studied, and they are not significant at this power level and length.

Another important issue in accelerator application is the trapped dipole mode that may cause beam instability. The number of SOL band gaps in the above PBG material is shown in Fig. 6. From the fundamental band gap to 4 times its frequency, about $6 \%$ of the spectrum space is covered by other band gaps, which makes it susceptible to trapping other defect modes. Numerical simulation has shown that is the case: some dipole modes are localized by the band gaps. Two factors contribute to this result: high mode density at higher frequency and wide band gaps. To remedy it, we also investigated another lattice, a so-called honeycomb lattice. It is formed by taking $1 / 3$ of the rods out of the existing triangular lattice, as shown in Fig. 7. At $r=0.24 a$, it has SOL band 


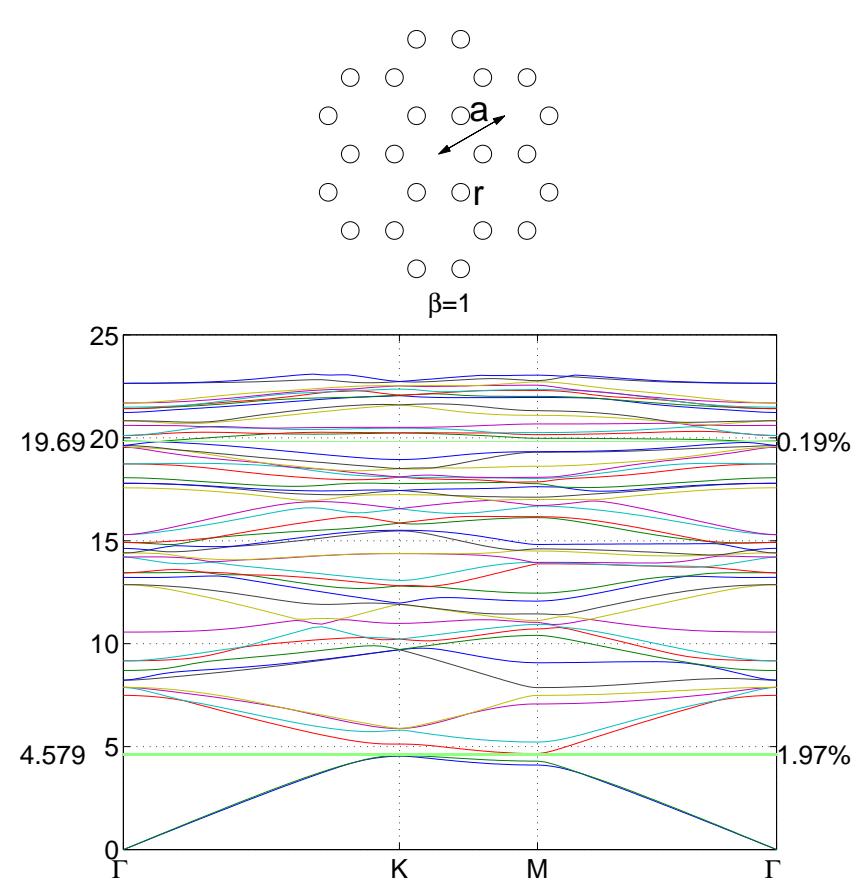

FIG. 7: Honeycomb lattice and its SOL band gap with $r / a=0.24$.

gaps at $k_{0}=4.579$ and 19.69 with the higher one extremely narrow. The lower frequency band gap localizes a monopole defect mode formed by replacing the center 6 air rods with a $r_{0}=0.19 a$ one, as illustrated in Fig. 8 . The defect mode has $k_{0}=k_{z}=4.56$. The simulation shows no trapped dipole modes in the lowest band gap. We did not check the higher band gap at 19.69 because of the computation time involved, but we believe it will not cause any adverse effect based on the band gap width.
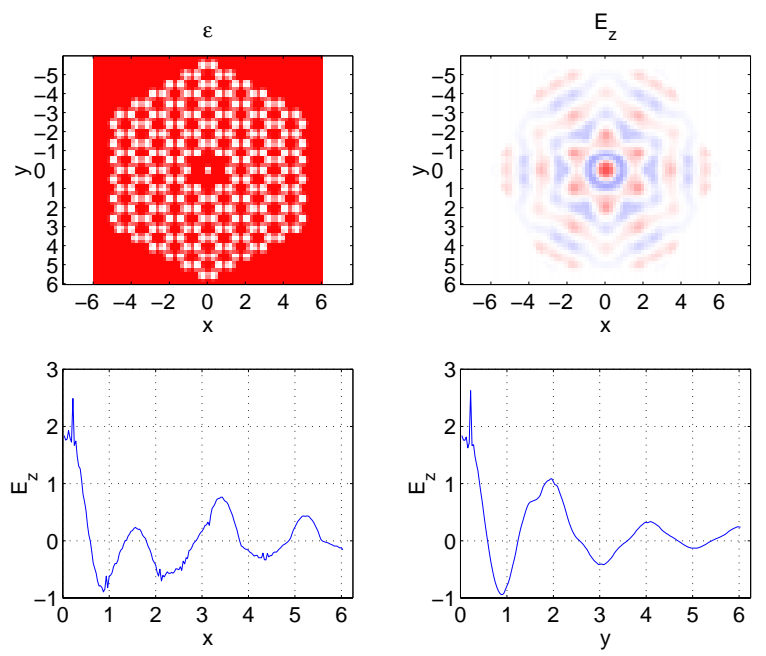

FIG. 8: Defect mode in honeycomb lattice.

Fused silica bundle like that in Fig. 4 has been manufactured with drawing method[7, 16]: Stacks of fiber tubes are bundled and repeatedly pulled down to desired dimensions. Pitch dimension $a$ from $53 \mathrm{~nm}$ to $2 \mu \mathrm{m}$ are achieved. Interstitial holes can be introduced by stacking cylindrical tubes instead of hexagon shaped canes. The multiple stack and draw process is especially suitable in massive production of PBGFA structures. The inexpensive manufacture process makes it possible to make multiple parallel linac bundles.

We have presented a schematic design of PBGFA structure that is able to reach $0.4 \mathrm{GeV} / \mathrm{m}$ gradient, and is relatively easy to manufacture. To our knowledge, this is the first numerical demonstration that 2D PBG supports an accelerator mode. With many advantages, PBGFA is a promising scheme that worth further investigations.

Even though we have demonstrated many aspect of PBGFA, the above examples are by no means optimal. More works need to be done on PBG material engineering that produces lower frequency SOL band gap and suitable gap/midgap ratio. Further more, defect mode with smaller field inside dielectric and higher beam loading are beneficial. Defect mode with quadrupole components to focus the electron beam is essential in maintaining beam stability and making parallel accelerator bundle possible. Pumping the tiny tubes to a high vacuum is another challenge. Further research into longer laser wavelength (for example, $10.6 \mu \mathrm{m}$ ) will benefit structure alignment, beam loading and manufacture tolerance, but its fate lies in the material's damage threshold.

The work is supported by DOE under contract No DEAC03-76SF00515.

\section{REFERENCES}

[1] E. Marcatili and R. Schmeltzer, The Bell System Technical Journal 43, 1783 (1964).

[2] M. E. Conde et al., in Advanced Accelerator Concepts Workshop, 8th, Baltimore, MD, 6-11 Jul 1998 (AIP, 1998), pp. 626-634.

[3] X. E. Lin et al., in preparation. .

[4] E. Yablonovitch, Phys. Rev. Lett. 58, 2059 (1987).

[5] S. John, Phys. Rev. Lett. 58, 2486 (1987).

[6] Y. Fink, D. J. Ripin, S. Fan, C. Chen, J. D. Joannopoulos, and E. L. Thomas, Journal of Lightwave Technology 17, 2039 (1999).

[7] R. Cregan, B.J.Mangan, J.C.Knight, T.A.Birks, P. Russell, P.J.Roberts, and D.C.Allan, Science 285, 1537 (1999).

[8] S. Lin, J. Fleming, D. Hetherington, B. Smith, R. Biswas, K. Ho, M. Sigalas, W. Zubrzycki, S. Kurtz, and J. Bur, Nature 396, 251 (1998).

[9] M. Plihal and A. Maradudin, Phys. Rev. B 44, 8565 (1991).

[10] R. Meade, K. Brommer, A. M. Rappe, and J. Joannopoulos, Appl. Phys. Lett. 61, 495 (1992).

[11] T. Birks, P. Roberts, P. Russell, D. Atkin, and T. Shepherd, Electronics Letters 31, 1941 (1995). 
[12] J. Knight, J. Broeng, T. Birks, and P. Russell, Science 282, 1476 (1998).

[13] K. Leung and Y. Liu, Phys. Rev. Lett. 65, 2646 (1990).

[14] B. C. Stuart, M. D. Feit, A. M. Rubenchik, B. W. Shore, and M. D. Perry, Phys. Rev. Lett. 74, 2248
(1995)

[15] P. Wilson, in Physics of High Energy Particle Accelerators, AIP Proc., 87 (AIP, 1981), pp. 450-563.

[16] R. J. Tonucci, B. L. Justus, A. J. Campillo, and C. E. Ford, Science 258, 783 (1992). 